\title{
Study of Low-altitude Slow and Small Target Detection on Radar
}

\author{
Daoming $\mathrm{Xu}^{1,}$, , Hongwei Zhang ${ }^{1}$ \\ ${ }^{1}$ Department of Electronic and Optics Engineering, Ordnance Engineering College, Shijiazhuang \\ 050003, China \\ axudaoming521@163.com
}

Keywords: LSS-target, Detection technology, Clutter suppression; Detection algorithm

\begin{abstract}
Holding low-altitude, slow and small (LSS) target has become a difficulty and study hotspot in air defense warning. This paper firstly introduces the characteristics of the LSS-target and the practical significance and military requirements for detecting such target, and briefly describes the difficulty of detecting radar targets. Then, finally, the prospects for the study of the LSS-target detection are presented.
\end{abstract}

\section{Introduction}

A Low-altitude slow and small (LSS) target refers to a variety of small aircraft and air drift, which have the characteristics of low altitude low altitude flight, flight speed is small and not easy to be detected[1].As the LSS target involves a wide range (including small and medium-sized aircraft, helicopters, gliders, delta, paragliding, parachute, hot air balloon, airship, UAV, air model, air balloons and other general aviation Equipment and aviation sports equipment, etc.), coupled with the development of science and technology, making the LSS target has been widely used and rapid development.

While the development of the LSS target has raised the level of national economic development in the civil sector, at the same time, its threats have become increasingly prominent to key objectives, key areas and major activities With the opening of China's low-altitude airspace, the LSS target monitored and prevented have become an urgent problem and it is also very important to detect, hurdle and against such targets.

The threat of LLS-target is even more pronounced in the military field. From several modern local war can be observed, low-altitude penetration has become one of the main means of combat. Because the low-altitude penetration weapons mostly fly below 600 meters, maneuverability, the use of terrain, the shading of objects, and strong ground / sea clutter and multipath effect of interference, making the target echo signal submerged in the strong sea / ground clutter, the air defense warning radar and other anti-aircraft weapons for its detection performance is greatly reduced.

In order to deal with the military and civilian threats caused by the LSS-target, how to effectively detect such target has become an important and hot research topic.

\section{Difficulties of the LSS-target detection}

The characteristics of LSS-target, such as low flight altitude, slow moving speed and small radar cross-section (RCS) make it difficult to detect them with a single technical means. The main problems faced by radar when detecting such targets:

1) Because of its low flying height, not only the detection background becomes complex and the target echo signal is strongly disturbed by the ground clutter, but also the target signal-to-noise ratio is greatly reduced. Since the clutter intensity is generally strong, the clutter must be suppressed firstly to ensure the target can be detected. The target has a serious overlap with the clutter spectrum in the Doppler domain. Therefore, it is difficult to avoid the suppression of the low-speed target signal while suppressing the clutter, resulting in the failure to detect the low-speed target. How to effectively suppress the clutter and detect the slow target becomes a key issue for detection of such target. 
2) Due to its low flight velocity, the echo signal is close to the zero frequency at the Doppler frequency domain and has strong overlap with the strong ground clutter and the slow clutter. It is difficult to detecting the target by the traditional filtering method in the frequency domain.

3) As the RCS is small, the echo energy is low and submerged in various clutter and noise. Because the target echo is weak, often submerged in the strong ground clutter, how to achieve the goal echo energy accumulation under the low signal-to-noise ratio background and to detect the LSS-target becomes badly important.

\section{Limitations of Existing Detection Techniques}

\section{Slow Target Detection Technologies.}

The traditional slow target detection methods are Kalmus filter and Clutter Map technique. Kalmus filter possesses certain clutter suppression ability and can keep the energy of low-speed target signal as far as possible, which can effectively improve the signal-to-clutter ratio of low-speed target.The Kalmus filter can be implemented by a discrete Fourier transform (DFT) equivalent comb filter [2]. Kalmus filter composition diagram shown in Figure 1.1Through the definition of DFT, we can deduce that DFT is equivalent to a filter bank with the response function (1-1)[2]:

$$
h_{k}(\mathrm{n})=\mathrm{e}^{-j \frac{2 \pi}{N}(\mathrm{~N}-1-\mathrm{n}) \mathrm{k}}
$$

Where $n=0,1 \cdots, N-1, \mathrm{~N}$ is the order of the filter, $0 \leq k \leq N-1$ is the group number of the filter.

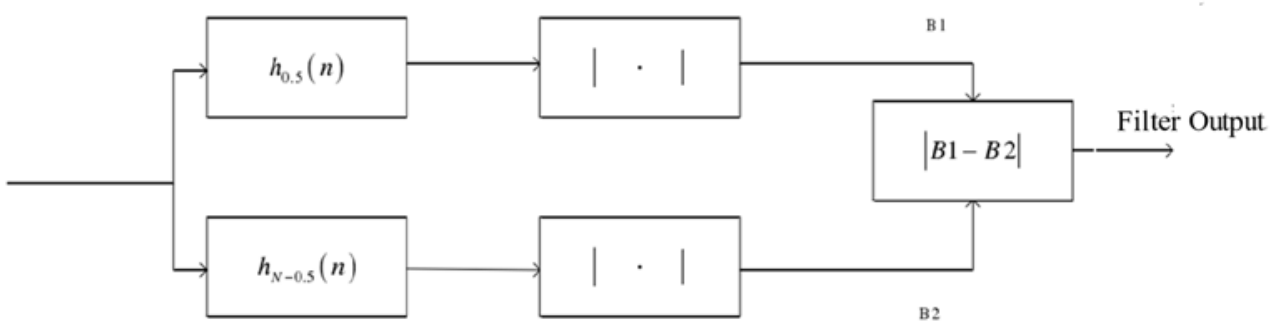

Figure 1.1 Kalmus filter composition diagram

Kalmus filter filtering process shown in Figure 1.1, the following steps:

1) The original input is first passed through the two filters $h_{0.5}(\mathrm{n})$ and $h_{N-0.5}(\mathrm{n})$. Where $k=0.5$ and $k=N-0.5$ are respectively substituted into the formula (1-1) to obtain the weight coefficients of the two filters $h_{0.5}(\mathrm{n})$ and $h_{\mathrm{N}-0.5}(\mathrm{n})$.

2) Then the output of the two filters were obtained by the absolute value of B1 and B2.

3) Finally, subtract B1 and B2 and then find the absolute value, the Kalmus filter output.

According to the Kalmus filter implementation process to find its amplitude response. Figure 1.2 shows the Kalmus filter block diagram of the two filters $h_{0.5}(\mathrm{n})$ and $h_{N-0.5}(\mathrm{n})$ spectrum, they are on the zero-frequency symmetry. To their amplitude-frequency response is subtracted, and then find the absolute value can be obtained Kalmus filter amplitude response. Through the above analysis, it is easy to find the DFT equivalent filter case Kalmus filter amplitude response (1-2):

$$
\left|H_{k a l}(\mathrm{f})\right|=\left\|H_{0}\left(\mathrm{f}-\frac{f_{r}}{2 N}\right)|-| H_{N-1}\left(\mathrm{f}-\frac{f_{r}}{2 N}\right)\right\|=\| \frac{\sin \left[\pi N\left(\frac{f}{f_{r}}-\frac{1}{2 N}\right)\right]}{\sin \left[\pi\left(\frac{f}{f_{r}}-\frac{1}{2 N}\right)\right]}|-| \frac{\sin \left[\pi N\left(\frac{f}{f_{r}}+\frac{1}{2 N}\right)\right]}{\sin \left[\pi\left(\frac{f}{f_{r}}+\frac{1}{2 N}\right)\right] \mid} \mid
$$


Where $\mathrm{f}$ is the sampling frequency. Figure 1.3 is a Kalmus filter frequency response diagram.

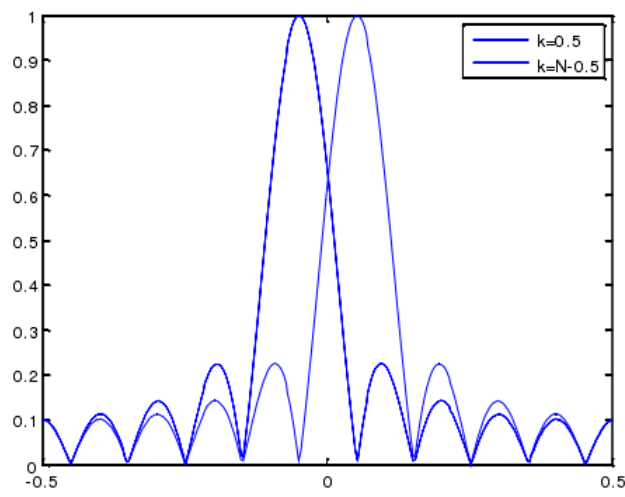

Figure 1.2 Kalmus filter block diagram of the two filters

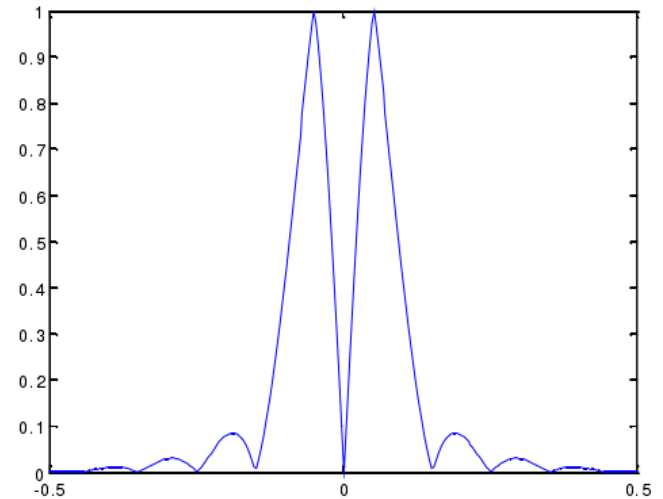

Figure 1.3 Kalmus filter frequency response diagram

Both the Kalmus filter and the MTI / MTD technique use different between clutter and target Doppler frequency to suppress the clutter through frequency domain filtering to improve the target detection performance. In order to further improve the radar ability to anti-clutter and detect low-speed targets, should also make full use of clutter characteristics.

Because clutter suppression can also suppress the energy of the low-speed target, the clutter pattern detection technique no longer suppresses the clutter, but estimates the clutter intensity through an inter-frame accumulation algorithm, and obtains the clutter intensity distribution within the range of radar Figure, used to set the detection threshold detection target. The method requires a high signal-to-noise ratio, otherwise the signal submerged in the clutter is difficult to detect. In addition, this method has the self-masking phenomenon, that is, when the target speed is too slow, the target signal does not fly out of the clutter unit after the multi-frame target, resulting in accumulation of the target signal into the clutter intensity estimation value.

The frequency resolution of modern spectral estimation method is expected to surpass the traditional spectral analysis method based on Fourier transform, which is very important for the study of slow target detection method. However, there is a problem of spectral splitting in AR spectrum estimation, while the Capon spectral performance is not estimation and its computation is complexity. In addition, assuming that the clutter spectrum is Gaussian and the center frequency is zero, the low-speed target detection problem can be regarded as a sine wave detected in Gaussian noise, so the super-resolution method can be used. However, due to the clutter spectrum is complex, the assumption is actually very different from the actual clutter, also used in the actual project difficultly. Empirical Mode Decomposition (EMD) technique, which can separate the clutter from the target signal, will decompose the false target and the computation is huge.

\section{Small Target Detection Technologies.}

The detection of small target in clutter background mainly has two aspects, one is how to suppress the strong clutter effectively, and the other is how to make the radar system detect the small target effectively. In the detection of radar small targets, we mainly rely on basic signal detection theory, such as hypothesis test theory, likelihood ratio test theory, Neyman-Pearson criterion [3], etc. Based on these mature theories, algorithm research and algorithm optimization has done a lot of work.

Liou R J [4] proposed a high-order correlation method using the correlation of the spatio-temporal domain to small targets from complex background clutter extracted, but in practice the application of a large amount of calculation. Blostein SD [5] and so on are based on the sequential hypothesis testing method, which belongs to the exhaustive searching method. All the possible trajectories in the image sequence are calculated. When the number of frames is short, the SNR is not enough. When the number is long, the number of trajectories is huge and there is no practical value.

In the domestic small target detection, Xiaoqiong Li of Beijing Institute of Technology [6] proposed a small target detection technique with high order cumulant, which has good resistance to background noise, improves the detection probability to a certain extent, Alarm probability. Yuexin Tian of the Institute of Optoelectronic Technology of North China [7] proposed a small target 
detection technique for Hough transform, which is easy to implement in algorithm and engineering, but only suitable for small signal detection under a certain signal-to-noise ratio.

\section{Conclusions}

The development of low and slow detection technology is very important for air defense security and people's livelihood. Based on the understanding of the characteristics of LSS-target and the limitations of the existing detection technology, this paper considers that the problem of LSS-target detection can be studied from the following points:

1) Clutter suppression is one of the key technologies in radar signal processing. The common clutter suppression technologies mainly include Moving Target Indication (MTI), Moving Target Detection (MTD), Pulse Doppler (PD) and so on. But in recent years the development of the drone technology and the stealth technology and the appearance of the slow small target make the separation of the target and the clutter be not ideal. So it is necessary to research the new clutter suppression technology.

2) Design specific test plans for specific targets. Aiming at different LSS-target, it is considered valid that a special detection scheme is designed to improve the detection probability by studying its echo characteristics.

3) Develop new systems of radar. The problem of the LSS-target detection is the inherent flaw of the existing radar system. To solve this problem, the improvement of the minutiae may not be enough. It is necessary to find the new method and the new idea to solve the problem by designing a new signal processing scheme.

\section{References}

[1] Chenyang Lu. Research on Methods for Low Speed Target Detection of Radar [D]. Xian: XiDian University, 2014.

[2] Xiaoyan Ma, Jiabin Xiang, Yusheng Zhu, Jiangmin Qin. Radar Signal Processing [M].Hunan. Hunan Science and Technology Press, 1998.

[3] Minxin Chen, Small Target Detection Under Sea Clutter [D], Xi'an University of Electronic Science and Technology, Xian, 2008, pp. 13-16.

[4] Ren Jean L., Mahmood R Dim., Target Detection Using High Order Correlation Method [J].IEEE Trans on AES.1993, 29 (3).841-856.

[5] Blostein S D, Richardson H S. A Sequential Detection Approach to Target Tracking [J].IEEE Trans on AES.1994, 30 (1), 197-212.

[6] Xiaoqiong Li, Caicheng Shi, Erke Mao et.al., Detection of infrared small targets based on higher order cumulants in single - frame complex cloud background [J]. Optical Technology. 2008, vol. 34, No. 5.

[7]YueXin Tian, Application of Hough Transform in Small Target Detection. Laser and infrared [J]. 2008, Vol. 38, No. 11. 